\title{
Dirichlet problem in Banach spaces: the bound sets approach
}

\author{
Jan Andres $^{1 *}$, Luisa Malaguti ${ }^{2}$ and Martina Pavlačková ${ }^{1}$
}

\author{
"Correspondence: \\ jan.andres@upol.cz \\ ${ }^{1}$ Department of Mathematical \\ Analysis, Faculty of Science, Palacký \\ University, 17. listopadu 12, \\ Olomouc, 771 46, Czech Republic \\ Full list of author information is \\ available at the end of the article
}

\begin{abstract}
The existence and localization result is obtained for a multivalued Dirichlet problem in a Banach space. The upper-Carathéodory and Marchaud right-hand sides are treated separately because in the latter case, the transversality conditions derived by means of bounding functions can be strictly localized on the boundaries of bound sets. MSC: 34A60; 34B15; 47H04

Keywords: Dirichlet problem; bounding functions; solutions in a given set; condensing multivalued operators
\end{abstract}

\section{Introduction}

The Dirichlet problem and its special case with homogeneous boundary conditions, usually called the Picard problem, belong to the most frequently studied boundary value problems. A lot of results concerning the standard problem for scalar second-order ordinary differential equations were generalized in various directions.

In Euclidean spaces, besides many extensions to vector equations, vector inclusions were under consideration, e.g., in [1-4]. In abstract spaces, usually in Banach and Hilbert spaces, equations, e.g., in [5-11] and inclusions, e.g., in $[9,12,13]$ were treated.

Sadovskii's or Darbo's fixed point theorems, jointly with the usage of a measure of noncompactness, were applied in $[5,8,9,11]$. Kakutani's or Ky Fan's fixed point theorems were applied with the upper and lower solutions technique in [9] and with a measure of noncompactness in [13]. On the other hand, continuation principles were employed in $[2,4,7]$.

The main aim of our present paper is an extension of the finite-dimensional results in $[2,4]$ into infinite-dimensional ones. We were also stimulated by the work of Jean Mawhin in [7], where degree arguments were applied to the Dirichlet problem in a Hilbert space probably for the first time, and in [14], where a bound sets approach was systematically developed. Hence, besides these two approaches, our extension consists in the consideration of differential inclusions in rather general Banach spaces and the usage of a measure of noncompactness. Similar results were already obtained in an analogous way by ourselves for Floquet problems in [15-18].

Besides the existence, the localization of solutions will be obtained in our main theorems (see Theorem 5.1 and Theorem 5.2). Unlike in [10], where the solutions belong to a positively invariant set, in our paper, some trajectories can escape from the prescribed set of candidate solutions. Moreover, the associated bound set need not be compact as in

(c) 2013 Andres et al.: licensee Springer. This is an Open Access article distributed under the terms of the Creative Commons Attribution License (http://creativecommons.org/licenses/by/2.0), which permits unrestricted use, distribution, and reproduction in any medium, provided the original work is properly cited. 
[10]. Similarly, the main difference between our results and those in $[9,13]$ consists in the application of a continuation principle jointly with a bound sets approach, which allows us to check fixed point free boundaries of given bound sets. This, in particular, means that, unlike in $[9,13]$, some trajectories can again escape from the prescribed set of candidate solutions in a transversal way.

Let $E$ be a Banach space (with the norm $\|\cdot\|$ ) satisfying the Radon-Nikodym property (e.g., reflexivity) and let us consider the Dirichlet boundary value problem (b.v.p.)

$$
\left.\begin{array}{l}
\ddot{x}(t) \in F(t, x(t), \dot{x}(t)) \quad \text { for a.a. } t \in[0, T], \\
x(0)=x(T)=0,
\end{array}\right\}
$$

where $F:[0, T] \times E \times E \multimap E$ is an upper-Carathéodory mapping or a globally upper semicontinuous mapping with compact, convex values (for the related definitions, see Section 2).

The main purpose of the present paper is to prove the existence of a Carathéodory solution $x \in A C^{1}([0, T], E)$ to problem (1) in a given set $Q$. This will be achieved by means of a suitable continuation principle. The crucial condition of the continuation principle described in Section 3 consists in guaranteeing the fixed point free boundary of $Q$ w.r.t. an admissible homotopical bridge starting from (1) (see condition (v) in Proposition 3.1 below). This requirement will be verified by means of Lyapunov-like bounding functions, i.e., via a bound sets technique. That is also why the whole Section 4 is devoted to this technique applied to Dirichlet problem (1). We will distinguish two cases, namely when $F$ is an upper-Carathéodory mapping and when $F$ is globally upper semicontinuous (i.e., a Marchaud mapping). Unlike in the first case, the second one allows us to apply bounding functions which can be strictly localized at the boundaries of given bound sets.

\section{Preliminaries}

Let $E$ be a Banach space having the Radon-Nikodym property (see, e.g., [19, pp.694-695]), i.e., if for every finite measure space $(\mathcal{M}, \Sigma, \mu)$ and every vector measure $m: \Sigma \rightarrow E$ of bounded variation, which is absolutely continuous w.r.t. $\mu$, we can find a Bochner integrable function $f: \mathcal{M} \rightarrow E$ such that

$$
m(C)=\int_{C} f(v) d \mu
$$

for each $C \in \Sigma$. Let $[0, T] \subset \mathbb{R}$ be a closed interval. By the symbol $L^{1}([0, T], E)$, we will mean the set of all Bochner integrable functions $x:[0, T] \rightarrow E$. For the definition and properties, see, e.g., [19, pp.693-701].

The symbol $A C^{1}([0, T], E)$ will denote the set of functions $x:[0, T] \rightarrow E$ whose first derivative $\dot{x}(\cdot)$ is absolutely continuous. Then $\ddot{x} \in L^{1}([0, T], E)$ and the fundamental theorem of calculus (the Newton-Leibniz formula) holds (see, e.g., [15, pp.243-244], [19, pp.695-696]). In the sequel, we will always consider $A C^{1}([0, T], E)$ as a subspace of the Banach space $C^{1}([0, T], E)$.

Given $C \subset E$ and $\varepsilon>0$, the symbol $B(C, \varepsilon)$ will denote, as usually, the set $C+\varepsilon B$, where $B$ is the open unit ball in $E$, i.e., $B=\{x \in E \mid\|x\|<1\}$.

We will also need the following definitions and notions from multivalued analysis. Let $X, Y$ be two metric spaces. We say that $F$ is a multivalued mapping from $X$ to $Y$ (written 
$F: X \multimap Y)$ if for every $x \in X$, a nonempty subset $F(x)$ of $Y$ is given. We associate with $F$ its graph $\Gamma_{F}$, the subset of $X \times Y$, defined by

$$
\Gamma_{F}:=\{(x, y) \in X \times Y \mid y \in F(x)\} .
$$

A multivalued mapping $F: X \multimap Y$ is called upper semicontinuous (shortly, u.s.c.) if for each open subset $U \subset Y$, the set $\{x \in X \mid F(x) \subset U\}$ is open in $X$.

A multivalued mapping $F: X \multimap Y$ is called compact if the set $F(X)=\bigcup_{x \in X} F(x)$ is contained in a compact subset of $Y$; it is called quasi-compact if it maps compact sets onto relatively compact sets; and completely continuous if it maps bounded sets onto relatively compact sets.

We say that a multivalued mapping $F:[0, T] \multimap Y$ with closed values is a step multivalued mapping if there exists a finite family of disjoint measurable subsets $I_{k}, k=1, \ldots, n$ such that $[0, T]=\bigcup I_{k}$ and $F$ is constant on every $I_{k}$. A multivalued mapping $F:[0, T] \multimap Y$ with closed values is called strongly measurable if there exists a sequence of step multivalued mappings $\left\{F_{n}\right\}$ such that $d_{H}\left(F_{n}(t), F(t)\right) \rightarrow 0$ as $n \rightarrow \infty$ for a.a. $t \in[0, T]$, where $d_{H}$ stands for the Hausdorff distance.

It is well known that if $Y$ is a Banach space, then a strongly measurable mapping $F:[0, T] \multimap Y$ with compact values possesses a single-valued strongly measurable selection (see, e.g., $[12,20])$.

A multivalued mapping $F:[0, T] \times X \multimap Y$ is called an upper-Carathéodory mapping if the map $F(\cdot, x):[0, T] \multimap Y$ is strongly measurable for all $x \in X$, the map $F(t, \cdot): X \multimap Y$ is u.s.c. for almost all $t \in[0, T]$ and the set $F(t, x)$ is compact and convex for all $(t, x) \in$ $[0, T] \times X$.

Let us note that if $X, Y$ are Banach spaces, then an upper-Carathéodory mapping $F:[0, T] \times X \multimap Y$ is weakly superpositionally measurable, i.e., that for each continuous $g:[0, T] \rightarrow X$, the composition $F(\cdot, g(\cdot)):[0, T] \multimap Y$ possesses a single-valued measurable selection (see, e.g., [12, 20]).

A multivalued mapping $F:[0, T] \times X \times X \multimap Y$ is called Lipschitzian in $(x, y) \in X \times X$ if there exists a constant $L>0$ such that

$$
d_{H}\left(F\left(t, x_{1}, y_{1}\right), F\left(t, x_{2}, y_{2}\right)\right) \leq L\left(\left\|x_{1}-x_{2}\right\|+\left\|y_{1}-y_{2}\right\|\right)
$$

for a.a. $t \in[0, T]$ and for all $x_{1}, x_{2}, y_{1}, y_{2} \in X$.

For more details concerning multivalued analysis, see, e.g., [12, 15, 20, 21].

In the sequel, the measure of noncompactness will also be employed.

Definition 2.1 Let $N$ be a partially ordered set, $E$ be a Banach space and let $P(E)$ denote the family of all nonempty subsets of $E$. A function $\beta: P(E) \rightarrow N$ is called a measure of noncompactness (m.n.c.) in $E$ if $\beta(\overline{c o \Omega})=\beta(\Omega)$ for all $\Omega \in P(E)$, where $\overline{c o \Omega}$ denotes the closed convex hull of $\Omega$.

An m.n.c. $\beta$ is called:

(i) monotone if $\beta\left(\Omega_{1}\right) \leq \beta\left(\Omega_{2}\right)$ for all $\Omega_{1} \subset \Omega_{2} \subset E$,

(ii) nonsingular if $\beta(\{x\} \cup \Omega)=\beta(\Omega)$ for all $x \in E$ and $\Omega \subset E$,

(iii) invariant with respect to the union with compact sets if $\beta(K \cup \Omega)=\beta(\Omega)$ for every relatively compact $K \subset E$ and every $\Omega \subset E$, 
(iv) regular when $\beta(\Omega)=0$ if and only if $\Omega$ is relatively compact,

(v) algebraically semi-additive if $\beta\left(\Omega_{1}+\Omega_{2}\right) \leq \beta\left(\Omega_{1}\right)+\beta\left(\Omega_{2}\right)$ for all $\Omega_{1}, \Omega_{2} \subset E$.

Definition 2.2 An m.n.c. $\beta$ with values in a cone of a Banach space has the semihomogeneity property if $\beta(t \Omega)=|t| \beta(\Omega)$ for all $t \in \mathbb{R}$ and all $\Omega \subset E$.

It is obvious that an m.n.c. which is invariant with respect to the union with compact sets is also nonsingular.

The typical example of an m.n.c. is the Hausdorff measure of noncompactness $\gamma$ defined, for all $\Omega \subset E$, by

$$
\gamma(\Omega):=\inf \left\{\varepsilon>0 \mid \exists x_{1}, \ldots, x_{n} \in E: \Omega \subset \bigcup_{i=1}^{n} B\left(\left\{x_{i}\right\}, \varepsilon\right)\right\} .
$$

The Hausdorff measure of noncompactness is monotone, nonsingular, algebraically semiadditive and has the semi-homogeneity property.

Let $\left\{f_{n}\right\} \subset L^{1}([0, T], E)$ be such that $\left\|f_{n}(t)\right\| \leq \alpha(t), \gamma\left(\left\{f_{n}(t)\right\}\right) \leq c(t)$ for a.a. $t \in[0, T]$, all $n \in \mathbb{N}$ and suitable $\alpha, c \in L^{1}([0, T], \mathbb{R})$, then $(c f .[20])$

$$
\gamma\left(\left\{\int_{0}^{T} f_{n}(t) d t\right\}\right) \leq 2 \int_{0}^{T} c(t) d t \quad \text { for a.a. } t \in[0, T] .
$$

Moreover, for all subsets $\Omega$ of $E$ (see, e.g., [18]),

$$
\gamma\left(\bigcup_{\lambda \in[0,1]} \lambda \Omega\right) \leq \gamma(\Omega)
$$

Let us now introduce the function

$$
\begin{aligned}
\mu(\Omega):= & \max _{\left\{w_{n}\right\}_{n} \subset \Omega}\left(\sup _{t \in[0, T]}\left[\gamma\left(\left\{w_{n}(t)\right\}_{n}\right)+\gamma\left(\left\{\dot{w}_{n}(t)\right\}_{n}\right)\right],\right. \\
& \left.\bmod _{C}\left(\left\{w_{n}\right\}_{n}\right)+\bmod _{C}\left(\left\{\dot{w}_{n}\right\}_{n}\right)\right),
\end{aligned}
$$

defined on the bounded set $\Omega \subset C^{1}([0, T], E)$, where the ordering is induced by the positive cone in $\mathbb{R}^{2}$ and where $\bmod _{C}(\Omega)$ denotes the modulus of continuity of a subset $\Omega \subset C([0, T], E))^{\text {a }}$ Such a $\mu$ is an m.n.c. in $C^{1}([0, T], E)$, as shown in the following lemma (proven in [16]), where the properties of $\mu$ will be also discussed.

Lemma 2.1 The function $\mu$ given by (4) defines an m.n.c. in $C^{1}([0, T], E)$; such an m.n.c. $\mu$ is monotone, invariant with respect to the union with compact sets and regular.

The m.n.c. $\mu$ defined by (4) will be used in order to solve problem (1) (cf. Theorem 5.1).

Definition 2.3 Let $E$ be a Banach space and $X \subset E$. A multivalued mapping $F: X \multimap E$ with compact values is called condensing with respect to an m.n.c. $\beta$ (shortly, $\beta$-condensing) if for every $\Omega \subset X$ such that $\beta(F(\Omega)) \geq \beta(\Omega)$, it holds that $\Omega$ is relatively compact.

A family of mappings $G: X \times[0,1] \multimap E$ with compact values is called $\beta$-condensing if for every $\Omega \subset X$ such that $\beta(G(\Omega \times[0,1])) \geq \beta(\Omega)$, it holds that $\Omega$ is relatively compact. 
The following convergence result will be also employed.

Lemma 2.2 (cf. [15, Lemma III.1.30]) Let E be a Banach space and assume that the sequence of absolutely continuous functions $x_{k}:[0, T] \rightarrow E$ satisfies the following conditions:

(i) the set $\left\{x_{k}(t) \mid k \in \mathbb{N}\right\}$ is relatively compact for every $t \in[0, T]$,

(ii) there exists $\alpha \in L^{1}([0, T],[0, \infty))$ such that $\left\|\dot{x}_{k}(t)\right\| \leq \alpha(t)$ for a.a. $t \in[0, T]$ and for all $k \in \mathbb{N}$,

(iii) the set $\left\{\dot{x}_{k}(t) \mid k \in \mathbb{N}\right\}$ is weakly relatively compact for a.a. $t \in[0, T]$.

Then there exists a subsequence of $\left\{x_{k}\right\}$ (for the sake of simplicity denoted in the same way as the sequence) converging to an absolutely continuous function $x:[0, T] \rightarrow$ E in the following way:

1. $\left\{x_{k}\right\}$ converges uniformly to $x$ in $C([0, T], E)$,

2. $\left\{\dot{x}_{k}\right\}$ converges weakly in $L^{1}([0, T], E)$ to $\dot{x}$.

The following lemma is well known when the Banach spaces $E_{1}$ and $E_{2}$ coincide (see, e.g., [22, p.88]). The present slight modification for $E_{1} \neq E_{2}$ was proved in [23].

Lemma 2.3 Let $[0, T] \subset \mathbb{R}$ be a compact interval, let $E_{1}, E_{2}$ be Banach spaces and let $F:[0, T] \times E_{1} \multimap E_{2}$ be a multivalued mapping satisfying the following conditions:

(i) $F(\cdot, x)$ has a strongly measurable selection for every $x \in E_{1}$,

(ii) $F(t, \cdot)$ is u.s.c. for a.a. $t \in[0, T]$,

(iii) the set $F(t, x)$ is compact and convex for all $(t, x) \in[0, T] \times E_{1}$.

Assume in addition that for every nonempty, bounded set $\Omega \subset E_{1}$, there exists $v=v(\Omega) \in$ $L^{1}([0, T],(0, \infty))$ such that

$$
\|F(t, x)\| \leq v(t)
$$

for a.a. $t \in[0, T]$ and every $x \in \Omega$. Let us define the Nemytskii operator $N_{F}: C\left([0, T], E_{1}\right) \multimap$ $L^{1}\left([0, T], E_{2}\right)$ in the following way: $N_{F}(x):=\left\{f \in L^{1}\left([0, T], E_{2}\right) \mid f(t) \in F(t, x(t))\right.$, a.e. on $[0, T]\}$ for every $x \in C\left([0, T], E_{1}\right)$. Then, if sequences $\left\{x_{k}\right\} \subset C\left([0, T], E_{1}\right)$ and $\left\{f_{k}\right\} \subset$ $L^{1}\left([0, T], E_{2}\right), f_{k} \in N_{F}\left(x_{k}\right), k \in \mathbb{N}$, are such that $x_{k} \rightarrow x$ in $C\left([0, T], E_{1}\right)$ and $f_{k} \rightarrow f$ weakly in $L^{1}\left([0, T], E_{2}\right)$, then $f \in N_{F}(x)$.

\section{Continuation principle}

The proof of the main result ( $c f$. Theorem 5.1 below) will be based on the combination of a bound sets technique together with the following continuation principle developed in [16].

Proposition 3.1 Let us consider the general multivalued b.v.p.

$$
\left.\begin{array}{l}
\ddot{x}(t) \in \varphi(t, x(t), \dot{x}(t)) \quad \text { for a.a. } t \in[0, T], \\
x \in S
\end{array}\right\}
$$

where $\varphi:[0, T] \times E \times E \multimap E$ is an upper-Carathéodory mapping and $S \subset A C^{1}([0, T], E)$. Let $H:[0, T] \times E \times E \times E \times E \times[0,1] \multimap E$ be an upper-Carathéodory mapping such that

$$
H(t, c, d, c, d, 1) \subset \varphi(t, c, d)
$$


for all $(t, c, d) \in[0, T] \times E \times E$. Moreover, assume that the following conditions hold:

(i) There exist a closed set $S_{1} \subset S$ and a closed, convex set $Q \subset C^{1}([0, T], E)$ with a nonempty interior Int $Q$ such that each associated problem

$$
\left.\begin{array}{l}
\ddot{x}(t) \in H(t, x(t), \dot{x}(t), q(t), \dot{q}(t), \lambda), \quad \text { for a.a. } t \in[0, T], \\
x \in S_{1},
\end{array}\right\}
$$

where $q \in Q$ and $\lambda \in[0,1]$, has a nonempty, convex set of solutions (denoted by $\mathfrak{T}(q, \lambda))$.

(ii) For every nonempty, bounded set $\Omega \subset E \times E$, there exists $v_{\Omega} \in L^{1}([0, T],[0, \infty))$ such that

$$
\|H(t, x, y, q(t), \dot{q}(t), \lambda)\| \leq v_{\Omega}(t)
$$

for a.a. $t \in[0, T]$ and all $(x, y) \in \Omega, q \in Q$ and $\lambda \in[0,1]$.

(iii) The solution mapping $\mathfrak{T}$ is quasi-compact and $\mu$-condensing with respect to $a$ monotone and nonsingular m.n.c. $\mu$ defined on $C^{1}([0, T], E)$.

(iv) For each $q \in Q$, the set of solutions of the problem $P(q, 0)$ is a subset of $\operatorname{Int} Q$, i.e., $\mathfrak{T}(q, 0) \subset$ Int $Q$ for all $q \in Q$.

(v) For each $\lambda \in(0,1)$, the solution mapping $\mathfrak{T}(\cdot, \lambda)$ has no fixed points on the boundary $\partial Q$ of $Q$.

Then the b.v.p. (5) has a solution in $Q$.

The proof of the continuation principle is based on the fact that the family $P(q, \lambda)$ of problems depending on two parameters $q \in Q$ and $\lambda \in[0,1]$ is associated to the original b.v.p. (5). This family is defined in such a way that if $\mathfrak{T}: Q \times[0,1] \multimap A C^{1}([0, T], E)$ is its corresponding solution mapping, then all fixed points of the map $\mathfrak{T}(\cdot, 1)$ are solutions of (5) (see condition (6)).

\section{Bound sets technique}

The continuation principle formulated in Proposition 3.1 requires, in particular, the existence of a suitable set $Q \subset A C^{1}([0, T], E)$ of candidate solutions. The set $Q$ should satisfy the transversality condition (v), i.e., it should have a fixed-point free boundary with respect to the solution mapping $\mathfrak{T}$. Since the direct verification of the transversality condition is usually a difficult task, we will devote this section to a bound sets technique which can be used for guaranteeing such a condition. For this purpose, we will define the set $Q$ as $Q:=C^{1}([0, T], \bar{K})$, where $K$ is nonempty and open in $E$ and $\bar{K}$ denotes its closure.

Hence, let us consider the Dirichlet boundary value problem (1) and let $V: E \rightarrow \mathbb{R}$ be a $C^{1}$-function satisfying

(H1) $\left.V\right|_{\partial K}=0$,

(H2) $V(x) \leq 0$ for all $x \in \bar{K}$.

Definition 4.1 A nonempty open set $K \subset E$ is called a bound set for the b.v.p. (1) if every solution $x$ of (1) such that $x(t) \in \bar{K}$ for each $t \in[0, T]$ does not satisfy $x\left(t^{*}\right) \in \partial K$ for any $t^{*} \in[0, T]$. 
Let $E^{\prime}$ be the Banach space dual to $E$ and let us denote by $\left.\langle\cdot, \cdot\rangle\right\rangle$ the pairing (the duality relation) between $E$ and $E^{\prime}$, i.e., for all $\Phi \in E^{\prime}$ and $x \in E$, we put $\Phi(x):=\langle\Phi, x\rangle$. The proof of the following proposition is quite analogous to the finite-dimensional case considered in [4]. Nevertheless, for the sake of completeness, we present it here, too.

Proposition 4.1 Let $K \subset E$ be an open set such that $0 \in K$ and $F:[0, T] \times E \times E \multimap E$ be an upper-Carathéodory mapping. Assume that the function $V \in C^{1}(E, \mathbb{R})$ has a locally Lipschitzian Fréchet derivative $\dot{V}_{x}$ and satisfies conditions $(\mathrm{H} 1)$ and $(\mathrm{H} 2)$. Suppose, moreover, that there exists $\varepsilon>0$ such that, for all $x \in \bar{K} \cap B(\partial K, \varepsilon), t \in(0, T)$ and $y \in E$, at least one of the following conditions:

$$
\begin{aligned}
& \limsup _{h \rightarrow 0^{-}} \frac{\left\langle\dot{V}_{x+h y}-\dot{V}_{x}, y\right\rangle}{h}+\left\langle\dot{V}_{x}, w\right\rangle>0, \\
& \limsup _{h \rightarrow 0^{+}} \frac{\left\langle\dot{V}_{x+h y}-\dot{V}_{x}, y\right\rangle}{h}+\left\langle\dot{V}_{x}, w\right\rangle>0
\end{aligned}
$$

holds for all $w \in F(t, x, y)$. Then $K$ is a bound set for the Dirichlet problem (1).

Proof Let $x:[0, T] \rightarrow \bar{K}$ be a solution of problem (1). We assume, by a contradiction, that there exists $t^{*} \in[0, T]$ such that $x\left(t^{*}\right) \in \partial K$. The point $t^{*}$ must lie in $(0, T)$ according to the Dirichlet boundary conditions and the fact that $0 \in K$.

Since $\dot{V}_{x}$ is locally Lipschitzian, there exist a neighborhood $U$ of $x\left(t^{*}\right)$ and a constant $L>0$ such that $\left.\dot{V}\right|_{U}$ is Lipschitzian with a constant $L$. Let $\delta>0$ be such that $x(t) \in U \cap B(\partial K, \varepsilon)$ for each $t \in\left[t^{*}-\delta, t^{*}+\delta\right]$.

In order to get the desired contradiction, let us define the function $g:[0, T] \rightarrow \mathbb{R}$ as the composition $g(t):=(V \circ x)(t)$. According to the regularity properties of $x$ and $V, g \in$ $C^{1}([0, T], \mathbb{R})$. Since $g\left(t^{*}\right)=0$ and $g(t) \leq 0$ for all $t \in[0, T], t^{*}$ is a local maximum point for $g$. Therefore, $\dot{g}\left(t^{*}\right)=0$. Moreover, there exist points $t^{* * *} \in\left(t^{*}-\delta, t^{*}\right), t^{* * *} \in\left(t^{*}, t^{*}+\delta\right)$ such that $\dot{g}\left(t^{* * *}\right) \geq 0$ and $\dot{g}\left(t^{* * *}\right) \leq 0$.

Since $\dot{g}(t)=\left\langle\dot{V}_{x(t)}, \dot{x}(t)\right\rangle$, where $\dot{V}_{x(t)}$ is locally Lipschitzian and $\dot{x}(t)$ is absolutely continuous on $\left[t^{*}-\delta, t^{*}\right], \ddot{g}(t)$ exists for a.a. $t \in\left[t^{*}-\delta, t^{*}+\delta\right]$. Consequently,

$$
0 \geq-\dot{g}\left(t^{* * *}\right)=\dot{g}\left(t^{*}\right)-\dot{g}\left(t^{* * *}\right)=\int_{t^{* *}}^{t^{*}} \ddot{g}(s) d s
$$

and

$$
0 \geq \dot{g}\left(t^{* * *}\right)=\dot{g}\left(t^{* * *}\right)-\dot{g}\left(t^{*}\right)=\int_{t^{*}}^{t^{* * * *}} \ddot{g}(s) d s
$$

At first, let us assume that condition (7) holds and let $t \in\left(t^{* *}, t^{*}\right)$ be such that $\ddot{g}(t)$ and $\ddot{x}(t)$ exist. Then

$$
\lim _{h \rightarrow 0} \frac{\dot{x}(t+h)-\dot{x}(t)}{h}=\ddot{x}(t),
$$

and so there exists a function $a(h), a(h) \rightarrow 0$ as $h \rightarrow 0$, such that for each $h$,

$$
\dot{x}(t+h)=\dot{x}(t)+h[\ddot{x}(t)+a(h)] .
$$


Moreover, since $x \in C^{1}([0, T], E)$, there exists a function $b(h), b(h) \rightarrow 0$ as $h \rightarrow 0$, such that for each $h$,

$$
x(t+h)=x(t)+h[\dot{x}(t)+b(h)] .
$$

Consequently, we obtain

$$
\begin{aligned}
\ddot{g}(t)= & \lim _{h \rightarrow 0} \frac{\dot{g}(t+h)-\dot{g}(t)}{h}=\limsup _{h \rightarrow 0^{-}} \frac{\dot{g}(t+h)-\dot{g}(t)}{h} \\
= & \limsup _{h \rightarrow 0^{-}} \frac{\left\langle\dot{V}_{x(t+h)} \dot{x}(t+h)\right\rangle-\left\langle\dot{V}_{x(t)}, \dot{x}(t)\right\rangle}{h} \\
= & \limsup _{h \rightarrow 0^{-}} \frac{\left\langle\dot{V}_{x(t)+h[\dot{x}(t)+b(h)]}, \dot{x}(t)+h[\ddot{x}(t)+a(h)]\right\rangle-\left\langle\dot{V}_{x(t)}, \dot{x}(t)\right\rangle}{h} \\
\geq & \limsup _{h \rightarrow 0^{-}} \frac{\left\langle\dot{V}_{x(t)+h \dot{x}(t),} \dot{x}(t)+h[\ddot{x}(t)+a(h)]\right\rangle-\left\langle\dot{V}_{x(t)}, \dot{x}(t)\right\rangle}{h} \\
& -L \cdot|b(h)| \cdot\|\dot{x}(t)+h[\ddot{x}(t)+a(h)]\| \\
= & \limsup _{h \rightarrow 0^{-}} \frac{\left\langle\dot{V}_{x(t)+h \dot{x}(t)}, \dot{x}(t)+h \ddot{x}(t)\right\rangle-\left\langle\dot{V}_{x(t)}, \dot{x}(t)\right\rangle}{h} \\
& -L \cdot|b(h)| \cdot\|\dot{x}(t)+h[\ddot{x}(t)+a(h)]\|+\left\langle\dot{V}_{x(t)+h \dot{x}(t), a(h)) .}\right.
\end{aligned}
$$

Since $\left\langle\dot{V}_{x(t)+h \dot{x}(t)}, a(h)\right\rangle-L \cdot|b(h)| \cdot\|\dot{x}(t)+h[\ddot{x}(t)+a(h)]\| \rightarrow 0$ as $h \rightarrow 0$,

$$
\begin{aligned}
\ddot{g}(t) & \geq \limsup _{h \rightarrow 0^{-}} \frac{\left\langle\dot{V}_{x(t)+h \dot{x}(t)}, \dot{x}(t)+h \ddot{x}(t)\right\rangle-\left\langle\dot{V}_{x(t)}, \dot{x}(t)\right\rangle}{h} \\
& =\limsup _{h \rightarrow 0^{-}} \frac{\left\langle\dot{V}_{x(t)+h \dot{x}(t)}-\dot{V}_{x(t)}, \dot{x}(t)\right\rangle}{h}+\left\langle\dot{V}_{x(t)+h \dot{x}(t)}, \ddot{x}(t)\right\rangle .
\end{aligned}
$$

Moreover, for every $x, w \in E$ and $h \in \mathbb{R}$, we have that

$$
\left\langle\dot{V}_{x+h y}, w\right\rangle=\left\langle\dot{V}_{x}, w\right\rangle+\left[\left\langle\dot{V}_{x+h y}, w\right\rangle-\left\langle\dot{V}_{x}, w\right\rangle\right]
$$

According to the Lipschitzianity of $\dot{V}$, when $|h|$ is sufficiently small, we have that

$$
\begin{aligned}
\left|\left\langle\dot{V}_{x+h y}, w\right\rangle-\left\langle\dot{V}_{x}, w\right\rangle\right| & =\left|\left\langle\dot{V}_{x+h y}-\dot{V}_{x}, w\right\rangle\right| \\
& \leq\left\|V_{x+h y}-\dot{V}_{x}\right\| \cdot\|w\| \leq L|h| \cdot\|y\| \cdot\|w\|,
\end{aligned}
$$

where $L$ denotes the local Lipschitz constant of $\dot{V}$ in a neighborhood of $x$. It implies that

$$
\lim _{h \rightarrow 0}\left\langle\dot{V}_{x+h y}, w\right\rangle-\left\langle\dot{V}_{x}, w\right\rangle=0
$$

and then

$$
\lim _{h \rightarrow 0}\left\langle\dot{V}_{x+h y}, w\right\rangle=\left\langle\dot{V}_{x}, w\right\rangle .
$$


Therefore,

$$
\ddot{g}(t) \geq \limsup _{h \rightarrow 0^{-}} \frac{\left\langle\dot{V}_{x(t)+h \dot{x}(t)}-\dot{V}_{x(t)}, \dot{x}(t)\right\rangle}{h}+\left\langle\dot{V}_{x(t)}, \ddot{x}(t)\right\rangle>0
$$

according to assumption (7), it leads to a contradiction with inequality (9).

Secondly, let us assume that condition (8) holds and let $s \in\left(t^{*}, t^{* * * *}\right)$ be such that $\ddot{g}(s)$ and $\ddot{x}(s)$ exist. Then it is possible to show, using the same procedure as before, that according to assumption (8),

$$
\ddot{g}(s) \geq \limsup _{h \rightarrow 0^{+}} \frac{\left\langle\dot{V}_{x(s)+h \dot{x}(s)}-\dot{V}_{x(s)}, \dot{x}(s)\right\rangle}{h}+\left\langle\dot{V}_{x(s)}, \ddot{x}(s)\right\rangle>0,
$$

which leads to a contradiction with inequality (10).

Therefore, we get the contradiction in case that at least one of conditions (7), (8) holds which completes the proof.

If the mapping $F(t, x, y)$ is globally u.s.c. in $(t, x, y)$, then the transversality conditions can be localized directly on the boundary of $K$, as will be shown in the following proposition, whose proof is again quite analogous to the finite-dimensional case considered in [2].

Proposition 4.2 Let $K \subset E$ be a nonempty open set such that $0 \in K$ and $F:[0, T] \times E \times$ $E \multimap$ E be an upper semicontinuous multivalued mapping with compact, convex values. Assume that there exists a function $V \in C^{1}(E, \mathbb{R})$ with a locally Lipschitzian Fréchet derivative $\dot{V}_{x}$ which satisfies conditions (H1) and (H2). Suppose, moreover, that for all $x \in \partial K$, $t \in(0, T)$ and $y \in E$ with

$$
\left\langle\dot{V}_{x}, y\right\rangle=0,
$$

the following condition holds:

$$
\liminf _{h \rightarrow 0} \frac{\left\langle\dot{V}_{x+h y}, y\right\rangle}{h}+\left\langle\dot{V}_{x}, w\right\rangle>0
$$

for all $w \in F(t, x, y)$. Then $K$ is a bound set for problem (1).

Proof Let $x:[0, T] \rightarrow \bar{K}$ be a solution of problem (1). We assume, by a contradiction, that there exists $t_{0} \in[0, T]$ such that $x\left(t_{0}\right) \in \partial K$. Since $0 \in K$ and $x$ satisfies Dirichlet boundary conditions, $t_{0} \in(0, T)$.

Let us define the function $g:\left[-t_{0}, T-t_{0}\right] \rightarrow(-\infty, 0]$ as the composition $g(h):=(V \circ$ $x)\left(t_{0}+h\right)$. Then $g(0)=0$ and $g(h) \leq 0$ for all $h \in\left[-t_{0}, T-t_{0}\right]$, i.e., there is a local maximum for $g$ at the point 0 , and so $\dot{g}(0)=\left\langle\dot{V}_{x\left(t_{0}\right)}, \dot{x}\left(t_{0}\right)\right\rangle=0$. Consequently, $v:=\dot{x}\left(t_{0}\right)$ satisfies condition (11).

Since $\dot{V}_{x}$ is locally Lipschitzian, there exist a neighborhood $U$ of $x\left(t_{0}\right)$ and a constant $L>0$ such that $\left.\dot{V}\right|_{U}$ is Lipschitzian with a constant $L$.

Let $\left\{h_{k}\right\}_{k=1}^{\infty}$ be an arbitrary decreasing sequence of positive numbers such that $h_{k} \rightarrow 0^{+}$ as $k \rightarrow \infty, x\left(t_{0}+h\right) \in U$ for all $h \in\left(0, h_{1}\right)$. 
Since $g(0)=0$ and $g(h) \leq 0$ for all $h \in\left(0, h_{k}\right]$, there exists, for each $k \in \mathbb{N}, h_{k}^{*} \in\left(0, h_{k}\right)$ such that $\dot{g}\left(h_{k}^{*}\right) \leq 0$.

Since $x \in C^{1}([0, T], E)$, for each $k \in \mathbb{N}$,

$$
x\left(t_{0}+h_{k}^{*}\right)=x\left(t_{0}\right)+h_{k}^{*}\left[\dot{x}\left(t_{0}\right)+b_{k}^{*}\right]
$$

where $b_{k}^{*} \rightarrow 0$ as $k \rightarrow \infty$.

Let

$$
\zeta:=\left\{\frac{\dot{x}\left(t_{0}+h_{k}^{*}\right)-\dot{x}\left(t_{0}\right)}{h_{k}^{*}}, k \in \mathbb{N}\right\}
$$

and let $\varepsilon>0$ be given. As a consequence of the regularity assumptions imposed on $F$ and of the continuity of both $x$ and $\dot{x}$, there exists $\bar{\delta}=\bar{\delta}(\varepsilon)>0$ such that for each $t \in(0, T)$, $\left|t-t_{0}\right| \leq \bar{\delta}$, it follows that

$$
F(t, x(t), \dot{x}(t)) \subset F\left(t_{0}, x\left(t_{0}\right), \dot{x}\left(t_{0}\right)\right)+\varepsilon \bar{B} .
$$

Subsequently, according to the mean-value theorem (see, e.g., [24, Theorem 0.5.3]), there exists $k_{\varepsilon} \in \mathbb{N}$ such that for each $k>k_{\varepsilon}$,

$$
\frac{\dot{x}\left(t_{0}+h_{k}^{*}\right)-\dot{x}\left(t_{0}\right)}{h_{k}^{*}}=\frac{1}{h_{k}^{*}} \int_{t_{0}}^{t_{0}+h_{k}^{*}} \ddot{x}(s) d s \in F\left(t_{0}, x\left(t_{0}\right), \dot{x}\left(t_{0}\right)\right)+\varepsilon \bar{B} .
$$

Therefore,

$$
\zeta \subset\left\{\frac{\dot{x}\left(t_{0}+h_{k}^{*}\right)-\dot{x}\left(t_{0}\right)}{h_{k}^{*}}, k=1,2, \ldots, k(\varepsilon)\right\} \cup F\left(t_{0}, x\left(t_{0}\right), \dot{x}\left(t_{0}\right)\right)+\varepsilon \bar{B} .
$$

Since $F$ has compact values and $\varepsilon$ is arbitrary, we obtain that $\zeta$ is a relatively compact set. Thus, there exist a subsequence, for the sake of simplicity denoted as the sequence, of $\left\{\frac{\dot{x}\left(t_{0}+h_{k}^{k}\right)-\dot{x}\left(t_{0}\right)}{h_{k}^{2}}\right\}$ and $w \in E$ such that

$$
\frac{\dot{x}\left(t_{0}+h_{k}^{*}\right)-\dot{x}\left(t_{0}\right)}{h_{k}^{*}} \rightarrow w
$$

as $k \rightarrow \infty$ implying, for the arbitrariness of $\varepsilon>0$,

$$
w \in F\left(t_{0}, x\left(t_{0}\right), \dot{x}\left(t_{0}\right)\right) \text {. }
$$

As a consequence of the property (14), there exists a sequence $\left\{a_{k}^{*}\right\}_{k=1}^{\infty}, a_{k}^{\prime \prime} \rightarrow 0$ as $k \rightarrow \infty$, such that

$$
\dot{x}\left(t_{0}+h_{k}^{*}\right)=\dot{x}\left(t_{0}\right)+h_{k}^{*}\left[w+a_{k}^{*}\right]
$$

for each $k \in \mathbb{N}$. Since $h_{k}^{*}>0$ and $\dot{g}\left(h_{k}^{*}\right) \leq 0$, in view of (13) and (15),

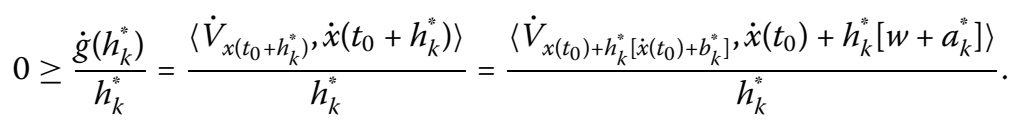


Since $h_{k}^{*} \in\left(0, h_{k}\right) \subset\left(0, h_{1}\right)$ for all $k \in \mathbb{N}$, we have, according to (13), that $x\left(t_{0}\right)+h_{k}^{\prime \prime}\left[\dot{x}\left(t_{0}\right)+\right.$ $\left.b_{k}^{*}\right] \in U$ for each $k \in \mathbb{N}$. Since $b_{k}^{*} \rightarrow 0$ as $k \rightarrow \infty$, it is possible to find $k_{0} \in \mathbb{N}$ such that for all $k \geq k_{0}$, it holds that $x\left(t_{0}\right)+\dot{x}\left(t_{0}\right) h_{k}^{*} \in U$. By means of the local Lipschitzianity of $\dot{V}$, for all $k \geq k_{0}$,

$$
\begin{aligned}
0 \geq & \frac{\dot{g}\left(h_{k}^{*}\right)}{h_{k}^{*}}=\frac{\left\langle\dot{V}_{x\left(t_{0}\right)+h_{k}^{*}\left[\dot{x}\left(t_{0}\right)+b_{k}^{*}\right]}-\dot{V}_{x\left(t_{0}\right)+h_{k}^{*} \dot{x}\left(t_{0}\right)}+\dot{V}_{x\left(t_{0}\right)+h_{k}^{*} \dot{x}\left(t_{0}\right)} \dot{x}\left(t_{0}\right)+h_{k}^{*}\left[w+a_{k}^{*}\right]\right\rangle}{h_{k}^{*}} \\
\geq & \frac{\left\langle\dot{V}_{x\left(t_{0}\right)+h_{k}^{*} \dot{x}\left(t_{0}\right)}, \dot{x}\left(t_{0}\right)+h_{k}^{*}\left[w+a_{k}^{*}\right]\right\rangle}{h_{k}^{*}}-L \cdot\left|b_{k}^{*}\right| \cdot\left\|\dot{x}\left(t_{0}\right)+h_{k}^{*}\left[w+a_{k}^{*}\right]\right\| \\
= & \frac{\left\langle\dot{V}_{x\left(t_{0}\right)+h_{k}^{*} \dot{x}\left(t_{0}\right)}, \dot{x}\left(t_{0}\right)+h_{k}^{*} w\right\rangle}{h_{k}^{*}}-L \cdot\left|b_{k}^{*}\right| \cdot\left\|\dot{x}\left(t_{0}\right)+h_{k}^{*}\left[w+a_{k}^{*}\right]\right\|+\left\langle\dot{V}_{x\left(t_{0}\right)+h_{k}^{*} \dot{x}\left(t_{0}\right)}, a_{k}^{*}\right\rangle \\
= & \frac{\left\langle\dot{V}_{x\left(t_{0}\right)+h_{k}^{*} \dot{x}\left(t_{0}\right)}, \dot{x}\left(t_{0}\right)\right\rangle}{h_{k}^{*}}+\left\langle\dot{V}_{x\left(t_{0}\right)}, w\right\rangle-L \cdot\left|b_{k}^{*}\right| \cdot\left\|\dot{x}\left(t_{0}\right)+h_{k}^{*}\left[w+a_{k}^{*}\right]\right\| \\
& +\left\langle\dot{V}_{x\left(t_{0}\right)+h_{k}^{*} \dot{x}\left(t_{0}\right)}, a_{k}^{*}\right\rangle .
\end{aligned}
$$

Since $\left\langle\dot{V}_{x\left(t_{0}\right)+h_{k}^{*} \dot{x}\left(t_{0}\right)}, a_{k}^{*}\right\rangle-L \cdot\left|b_{k}^{*}\right| \cdot\left\|\dot{x}\left(t_{0}\right)+h_{k}^{*}\left[w+a_{k}^{*}\right]\right\| \rightarrow 0$ as $k \rightarrow \infty$,

$$
\liminf _{h \rightarrow 0^{+}} \frac{\left\langle\dot{V}_{x\left(t_{0}\right)+h_{k}^{*} \dot{x}\left(t_{0}\right)}, \dot{x}\left(t_{0}\right)\right\rangle}{h_{k}^{*}}+\left\langle\dot{V}_{x\left(t_{0}\right)}, w\right\rangle \leq 0 .
$$

If we consider, instead of the sequence $\left\{h_{k}\right\}_{k=1}^{\infty}$, an increasing sequence $\left\{\bar{h}_{k}\right\}_{k=1}^{\infty}$ of negative numbers such that $\bar{h}_{k} \rightarrow 0^{-}$as $k \rightarrow \infty, x\left(t_{0}+h\right) \in U$ for all $h \in\left(\bar{h}_{1}, 0\right)$, we are able to find, for each $k \in \mathbb{N}, \bar{h}_{k}^{*} \in\left(\bar{h}_{k}, 0\right)$ such that $\dot{g}\left(\bar{h}_{k}^{*}\right) \geq 0$. Therefore, using the same procedure as in the first part of the proof, we obtain, for $k \in \mathbb{N}$ sufficiently large, that

$$
\begin{aligned}
0 \geq \frac{\dot{g}\left(\bar{h}_{k}^{*}\right)}{\bar{h}_{k}^{*}} \geq & \frac{\left\langle\dot{V}_{x\left(t_{0}\right)+\bar{h}_{k}^{*} \dot{x}\left(t_{0}\right)}, \dot{x}\left(t_{0}\right)\right\rangle}{\bar{h}_{k}^{*}} \\
& +\left\langle\dot{V}_{x\left(t_{0}\right)}, \bar{w}\right\rangle-L \cdot\left|\bar{b}_{k}^{*}\right| \cdot\left\|\dot{x}\left(t_{0}\right)+\bar{h}_{k}^{*}\left[\bar{w}+\bar{a}_{k}^{*}\right]\right\|+\left\langle\dot{V}_{x\left(t_{0}\right)+\bar{h}_{k}^{*} \dot{x}\left(t_{0}\right)}, \bar{a}_{k}^{*}\right\rangle,
\end{aligned}
$$

where $\bar{a}_{k}^{*} \rightarrow 0, \bar{b}_{k}^{*} \rightarrow 0$ as $k \rightarrow \infty$ and $\bar{w} \in F\left(t_{0}, x\left(t_{0}\right), \dot{x}\left(t_{0}\right)\right)$.

This means that $\left\langle\dot{V}_{x\left(t_{0}\right)+\bar{h}_{k}^{*} \dot{x}\left(t_{0}\right)}, \bar{a}_{k}^{*}\right\rangle-L \cdot\left|\bar{b}_{k}^{*}\right| \cdot\left\|\dot{x}\left(t_{0}\right)+\bar{h}_{k}^{*}\left[\bar{w}+\bar{a}_{k}^{*}\right]\right\| \rightarrow 0$ as $k \rightarrow \infty$, which implies

$$
\liminf _{h \rightarrow 0^{-}} \frac{\left\langle\dot{V}_{x\left(t_{0}\right)+h \dot{x}\left(t_{0}\right)}, \dot{x}\left(t_{0}\right)\right\rangle}{h}+\left\langle\dot{V}_{x\left(t_{0}\right)}, \bar{w}\right\rangle \leq 0 .
$$

Inequalities (16) and (17) are in a contradiction with condition (12), because $x\left(t_{0}\right) \in \partial K$, $\dot{x}\left(t_{0}\right)$ satisfies condition (11) and $w, \bar{w} \in F\left(t_{0}, x\left(t_{0}\right), \dot{x}\left(t_{0}\right)\right)$.

Remark 4.1 One can readily check that for $V \in C^{2}(E, \mathbb{R})$, inequalities (7) and (8), as well as (12), become

$$
\left\langle\ddot{V}_{x}(y), y\right\rangle+\left\langle\dot{V}_{x}, w\right\rangle>0
$$

with $t, x, y, w$ as in Proposition 4.1 or in Proposition 4.2. 
The typical case occurs when $E=H$ is a Hilbert space, $\langle$,$\rangle denotes the scalar product$ and

$$
V(x):=\frac{1}{2}\left(\|x\|^{2}-R^{2}\right)=\frac{1}{2}\left(\langle x, x\rangle-R^{2}\right)
$$

for some $R>0$. In this case, $V \in C^{2}(H, \mathbb{R})$ and it is not difficult to see that conditions (7) and (8), as well as (12), become

$$
\langle y, y\rangle+\langle x, w\rangle>0
$$

with $t, x, y$ and $w$ as in Proposition 4.1 or in Proposition 4.2, where $K:=\{x \in H \mid\|x\|<R\}$.

Definition 4.2 A $C^{1}$-function $V: E \rightarrow R$ with a locally Lipschitzian Fréchet derivative $\dot{V}$ which satisfies conditions (H1), (H2) and all assumptions in Proposition 4.1 or Proposition 4.2 is called a bounding function for problem (1).

\section{Existence and localization results}

Combining the continuation principle with the bound sets technique, we are ready to state the main result of the paper concerning the solvability and localization of a solution of the multivalued Dirichlet problem (1).

Theorem 5.1 Consider the Dirichlet b.v.p. (1), where F: [0,T] $\times E \times E \multimap E$ is an upperCarathéodory multivalued mapping. Assume that $K \subset E$ is an open, convex set containing 0. Furthermore, let the following conditions be satisfied:

$\left(5_{i}\right) \quad \gamma\left(F\left(t, \Omega_{1} \times \Omega_{2}\right)\right) \leq g(t)\left(\gamma\left(\Omega_{1}\right)+\gamma\left(\Omega_{2}\right)\right)$ for a.a. $t \in[0, T]$ and each bounded $\Omega_{1}, \Omega_{2} \subset$ $E$, where $g \in L^{1}([0, T],[0, \infty))$ and $\gamma$ is the Hausdorff measure of noncompactness in $E$.

$\left(5_{i i}\right)$ For every nonempty, bounded set $\Omega \subset E \times E$, there exists $v_{\Omega} \in L^{1}([0, T],[0, \infty))$ such that

$$
\|F(t, x, y)\| \leq v_{\Omega}(t)
$$

for a.a. $t \in[0, T]$ and all $(x, y) \in \Omega$,

$\left(5_{i i i}\right)(T+4)\|g\|_{L^{1}([0, T],[0, \infty))}<2$.

Finally, let there exist a function $V \in C^{1}(E, \mathbb{R})$ with a locally Lipschitzian Fréchet derivative $\dot{V}$ satisfying conditions $(\mathrm{H} 1),(\mathrm{H} 2)$, and at least one of conditions $(7),(8)$ for a suitable $\varepsilon>0$, all $x \in \bar{K} \cap B(\partial K, \varepsilon), t \in(0, T), y \in E, \lambda \in(0,1)$ and $w \in \lambda F(t, x, y)$. Then the Dirichlet b.v.p. (1) admits a solution whose values are located in $\bar{K}$.

Proof Let us define the closed set $S=S_{1}$ by

$$
S:=\left\{x \in A C^{1}([0, T], E): x(T)=x(0)=0\right\}
$$

and let the set $Q$ of candidate solutions be defined as $Q:=C^{1}([0, T], \bar{K})$. Because of the convexity of $K$, the set $Q$ is closed and convex. 
For all $q \in Q$ and $\lambda \in[0,1]$, consider still the associated fully linearized problem

$$
\left.\begin{array}{l}
\ddot{x}(t) \in \lambda F(t, q(t), \dot{q}(t)) \quad \text { for a.a. } t \in[0, T], \\
x(T)=x(0)=0,
\end{array}\right\}
$$

and denote by $\mathfrak{T}$ a solution mapping which assigns to each $(q, \lambda) \in Q \times[0,1]$ the set of solutions of $P(q, \lambda)$. We will show that the family of the above b.v.p.s $P(q, \lambda)$ satisfies all assumptions of Proposition 3.1.

In this case, $\varphi(t, x, \dot{x})=F(t, x, \dot{x})$ which, together with the definition of $P(q, \lambda)$, ensures the validity of (6).

ad (i) In order to verify condition (i) in Proposition 3.1, we need to show that for each $(q, \lambda) \in Q \times[0,1]$, the problem $P(q, \lambda)$ is solvable with a convex set of solutions. So, let $(q, \lambda) \in Q \times[0,1]$ be arbitrary and let $f_{q}(\cdot)$ be a strongly measurable selection of $F(\cdot, q(\cdot), \dot{q}(\cdot))$. The homogeneous problem corresponding to b.v.p. $P(q, \lambda)$,

$$
\left.\begin{array}{l}
\ddot{x}(t)=0 \quad \text { for a.a. } t \in[0, T] \\
x(T)=x(0)=0,
\end{array}\right\}
$$

has only the trivial solution, and therefore the single-valued Dirichlet problem

$$
\left.\begin{array}{l}
\ddot{x}(t)=\lambda f_{q}(t) \quad \text { for a.a. } t \in[0, T], \\
x(T)=x(0)=0
\end{array}\right\}
$$

admits a unique solution $x_{q, \lambda}(\cdot)$ which is one of solutions of $P(q, \lambda)$. This is given, for a.a. $t \in[0, T]$, by $x_{q, \lambda}(t)=\int_{0}^{T} G(t, s) \lambda f_{q}(s) d s$, where $G$ is the Green function associated to the homogeneous problem (19). The Green function $G$ and its partial derivative $\frac{\partial}{\partial t} G$ are defined by (cf., e.g., [12, pp.170-171])

$$
\begin{gathered}
G(t, s)= \begin{cases}\frac{(s-T) t}{T} & \text { for all } 0 \leq t \leq s \leq T, \\
\frac{(t-T) s}{T} & \text { for all } 0 \leq s \leq t \leq T,\end{cases} \\
\frac{\partial}{\partial t} G(t, s)= \begin{cases}\frac{(s-T)}{T} & \text { for all } 0 \leq t \leq s \leq T, \\
\frac{s}{T} & \text { for all } 0 \leq s \leq t \leq T .\end{cases}
\end{gathered}
$$

Thus, the set of solutions of $P(q, \lambda)$ is nonempty. The convexity of the solution sets follows immediately from the properties of a mapping $F$ and the fact that problems $P(q, \lambda)$ are fully linearized.

ad (ii) Assuming that $H:[0, T] \times E \times E \times E \times E \times[0,1] \multimap E$ is defined by $H(t, x, y$, $q, r, \lambda):=\lambda F(t, q, r)$, condition (ii) in Proposition 3.1 is ensured directly by assumption $\left(5_{i i}\right)$.

ad (iii) Since the verification of condition (iii) in Proposition 3.1 is technically the most complicated, it will be subdivided into two parts: (iii ${ }_{1}$ ) the quasi-compactness of the solution operator $\mathfrak{T},\left(\mathrm{iii}_{2}\right)$ the condensity of $\mathfrak{T}$ w.r.t. the monotone and nonsingular (cf. Lemma 2.1) m.n.c. $\mu$ defined by (4).

ad (iii $1_{1}$ ) Let us firstly prove that the solution mapping $\mathfrak{T}$ is quasi-compact. Since $C^{1}([0, T], E)$ is a metric space, it is sufficient to prove the sequential quasi-compactness of $\mathfrak{T}$. Hence, let us consider the sequences $\left\{q_{n}\right\},\left\{\lambda_{n}\right\}, q_{n} \in Q, \lambda_{n} \in[0,1]$ for all $n \in \mathbb{N}$ such 
that $q_{n} \rightarrow q$ in $C^{1}([0, T], E)$ and $\lambda_{n} \rightarrow \lambda$. Moreover, let $x_{n} \in \mathfrak{T}\left(q_{n}, \lambda_{n}\right)$ for all $n \in \mathbb{N}$. Then there exists, for all $n \in \mathbb{N}, f_{n}(\cdot) \in F\left(\cdot, q_{n}(\cdot), \dot{q}_{n}(\cdot)\right)$ such that

$$
\ddot{x}_{n}(t)=\lambda_{n} f_{n}(t) \quad \text { for a.a. } t \in[0, T]
$$

and that $x_{n}(T)=x_{n}(0)=0$.

Since $q_{n} \rightarrow q$ and $\dot{q}_{n} \rightarrow \dot{q}$ in $C([0, T], E)$, there exists a bounded $\Omega \subset E \times E$ such that $\left(q_{n}(t), \dot{q}_{n}(t)\right) \in \Omega$ for all $t \in[0, T]$ and $n \in \mathbb{N}$. Therefore, there exists, according to condition $\left(5_{i i}\right), v_{\Omega} \in L^{1}([0, T],[0, \infty))$ such that $\left\|f_{n}(t)\right\| \leq v_{\Omega}(t)$ for every $n \in \mathbb{N}$ and a.a. $t \in[0, T]$.

Moreover, for every $n \in \mathbb{N}$ and a.a. $t \in[0, T]$,

$$
x_{n}(t)=\lambda_{n} \int_{0}^{T} G(t, s) f_{n}(s) d s
$$

and

$$
\dot{x}_{n}(t)=\lambda_{n} \int_{0}^{T} \frac{\partial}{\partial t} G(t, s) f_{n}(s) d s
$$

Thus, $x_{n}$ satisfies, for every $n \in \mathbb{N}$ and a.a. $t \in[0, T],\left\|x_{n}(t)\right\| \leq a$ and $\left\|\dot{x}_{n}(t)\right\| \leq b$, where

$$
a:=\frac{T}{4} \int_{0}^{T} v_{\Omega}(s) d s
$$

and

$$
b:=\int_{0}^{T} v_{\Omega}(s) d s
$$

Furthermore, for every $n \in \mathbb{N}$ and a.a. $t \in[0, T]$, we have

$$
\left\|\ddot{x}_{n}(t)\right\| \leq v_{\Omega}(t) .
$$

Hence, the sequences $\left\{x_{n}\right\}$ and $\left\{\dot{x}_{n}\right\}$ are bounded and $\left\{\ddot{x}_{n}\right\}$ is uniformly integrable.

Since the sequences $\left\{q_{n}\right\},\left\{\dot{q}_{n}\right\}$ are converging, we obtain, in view of $\left(5_{i}\right)$,

$$
\gamma\left(\left\{f_{n}(t)\right\}\right) \leq g(t)\left(\gamma\left(\left\{q_{n}(t)\right\}\right)+\gamma\left(\left\{\dot{q}_{n}(t)\right\}\right)\right)=0
$$

for a.a. $t \in[0, T]$, which implies that $\left\{f_{n}(t)\right\}$ is relatively compact.

For all $(t, s) \in[0, T] \times[0, T]$, the sequence $\left\{G(t, s) f_{n}(s)\right\}$ is relatively compact as well since, according to the semi-homogeneity of the Hausdorff m.n.c.,

$$
\gamma\left(\left\{G(t, s) f_{n}(s)\right\}\right) \leq|G(t, s)| \gamma\left(\left\{f_{n}(s)\right\}\right)=0 \quad \text { for all }(t, s) \in[0, T] \times[0, T] .
$$

Moreover, by means of (2), (3), (21) and the semi-homogeneity of the Hausdorff m.n.c.,

$$
\gamma\left(\left\{x_{n}(t)\right\}\right) \leq \gamma\left(\bigcup_{\lambda \in[0,1]} \lambda\left\{\int_{0}^{T} G(t, s) f_{n}(s) d s\right\}\right) \leq \gamma\left(\left\{\int_{0}^{T} G(t, s) f_{n}(s) d s\right\}\right)=0 .
$$


By similar reasonings, we can also get

$$
\gamma\left(\left\{\dot{x}_{n}(t)\right\}\right)=0,
$$

by which $\left\{x_{n}(t)\right\},\left\{\dot{x}_{n}(t)\right\}$ are relatively compact for a.a. $t \in[0, T]$. Moreover, since $x_{n}$ satisfies for all $n \in \mathbb{N}$ equation (20), $\left\{\ddot{x}_{n}(t)\right\}$ is relatively compact for a.a. $t \in[0, T]$. Thus, according to Lemma 2.2, there exist a subsequence of $\left\{\dot{x}_{n}\right\}$, for the sake of simplicity denoted in the same way as the sequence, and $x \in C^{1}([0, T], E)$ such that $\left\{\dot{x}_{n}\right\}$ converges to $\dot{x}$ in $C([0, T], E)$ and $\left\{\ddot{x}_{n}\right\}$ converges weakly to $\ddot{x}$ in $L^{1}([0, T], E)$. Therefore, the mapping $\mathfrak{T}$ is quasi-compact.

ad $\left(\mathrm{iii}_{2}\right)$ In order to show that $\mathfrak{T}$ is $\mu$-condensing, where $\mu$ is defined by (4), we will prove that any bounded subset $\Theta \subset Q$ such that $\mu(\mathfrak{T}(\Theta \times[0,1])) \geq \mu(\Theta)$ is relatively compact. Let $\left\{x_{n}\right\}_{n} \subset \mathfrak{T}(\Theta \times[0,1])$ be a sequence such that

$$
\begin{aligned}
\mu & (\mathfrak{T}(\Theta \times[0,1])) \\
& =\left(\sup _{t \in[0, T]}\left[\gamma\left(\left\{x_{n}(t)\right\}_{n}\right)+\gamma\left(\left\{\dot{x}_{n}(t)\right\}_{n}\right)\right], \bmod _{C}\left(\left\{x_{n}\right\}_{n}\right)+\bmod _{C}\left(\left\{\dot{x}_{n}\right\}_{n}\right)\right) .
\end{aligned}
$$

Then we can find $\left\{q_{n}\right\}_{n} \subset \Theta,\left\{f_{n}\right\}_{n}$ satisfying $f_{n}(t) \in F\left(t, q_{n}(t), \dot{q}_{n}(t)\right)$ for a.a. $t \in[0, T]$ and $\left\{\lambda_{n}\right\}_{n} \subset[0,1]$ such that for all $t \in[0, T]$,

$$
x_{n}(t)=\lambda_{n} \int_{0}^{T} G(t, s) f_{n}(s) d s
$$

and

$$
\dot{x}_{n}(t)=\lambda_{n} \int_{0}^{T} \frac{\partial}{\partial t} G(t, s) f_{n}(s) d s
$$

In view of $\left(5_{i}\right)$, we have, for all $t \in[0, T]$,

$$
\begin{aligned}
& \gamma\left(\left\{f_{n}(t), n \in \mathbb{N}\right\}\right) \\
& \quad \leq g(t)\left(\gamma\left(\left\{q_{n}(t), n \in \mathbb{N}\right\}\right)+\gamma\left(\left\{\dot{q}_{n}(t), n \in \mathbb{N}\right\}\right)\right) \\
& \quad \leq g(t) \sup _{t \in[0, T]}\left(\gamma\left(\left\{q_{n}(t), n \in \mathbb{N}\right\}\right)+\gamma\left(\left\{\dot{q}_{n}(t), n \in \mathbb{N}\right\}\right)\right) .
\end{aligned}
$$

Since $\left\{q_{n}\right\}_{n} \subset \Theta$ and $\Theta$ is bounded in $C^{1}([0, T], E)$, by means of $\left(5_{i i}\right)$, we get the existence of $v_{\Theta} \in L^{1}([0, T],[0, \infty))$ such that $\left\|f_{n}(t)\right\| \leq v_{\Theta}(t)$ for a.a. $t \in[0, T]$ and all $n \in \mathbb{N}$. This implies $\left\|G(t, s) f_{n}(t)\right\| \leq|G(t, s)| v_{\Theta}(t)$ for a.a. $t, s \in[0, T]$ and all $n \in \mathbb{N}$.

Moreover, by virtue of the semi-homogeneity of the Hausdorff m.n.c., for all $(t, s) \in$ $[0, T] \times[0, T]$, we have

$$
\begin{aligned}
& \gamma\left(\left\{G(t, s) f_{n}(s), n \in \mathbb{N}\right\}\right) \\
& \quad \leq|G(t, s)| \gamma\left(\left\{f_{n}(s), n \in \mathbb{N}\right\}\right) \leq \frac{T}{4} \gamma\left(\left\{f_{n}(s), n \in \mathbb{N}\right\}\right) \\
& \quad \leq \frac{T}{4} g(t) \sup _{t \in[0, T]}\left(\gamma\left(\left\{q_{n}(t), n \in \mathbb{N}\right\}\right)+\gamma\left(\left\{\dot{q}_{n}(t), n \in \mathbb{N}\right\}\right)\right) .
\end{aligned}
$$


According to (2), (3) and (22), we so obtain for each $t \in[0, T]$,

$$
\begin{aligned}
\gamma\left(\left\{x_{n}(t), n \in \mathbb{N}\right\}\right) & \leq \gamma\left(\left\{\int_{0}^{T} G(t, s) f_{n}(s) d s, n \in \mathbb{N}\right\}\right) \\
& \leq 2 \frac{T}{4}\|g\|_{L^{1}} \sup _{t \in[0, T]}\left(\gamma\left(\left\{q_{n}(t), n \in \mathbb{N}\right\}\right)+\gamma\left(\left\{\dot{q}_{n}(t), n \in \mathbb{N}\right\}\right)\right) \\
& =\frac{T}{2}\|g\|_{L^{1}} \mathcal{S},
\end{aligned}
$$

where

$$
\mathcal{S}:=\sup _{t \in[0, T]}\left(\gamma\left(\left\{q_{n}(t), n \in \mathbb{N}\right\}\right)+\gamma\left(\left\{\dot{q}_{n}(t), n \in \mathbb{N}\right\}\right)\right) .
$$

By the similar reasonings, we can obtain that for each $t \in[0, T]$,

$$
\gamma\left(\left\{\dot{x}_{n}(t), n \in \mathbb{N}\right\}\right) \leq 2\|g\|_{L^{1}} \mathcal{S},
$$

when starting from condition (23). Subsequently,

$$
\gamma\left(\left\{x_{n}(t), n \in \mathbb{N}\right\}\right)+\gamma\left(\left\{\dot{x}_{n}(t), n \in \mathbb{N}\right\}\right) \leq \frac{T+4}{2}\|g\|_{L^{1}} \mathcal{S},
$$

yielding

$$
\sup _{t \in[0, T]}\left(\gamma\left(\left\{x_{n}(t), n \in \mathbb{N}\right\}\right)+\gamma\left(\left\{\dot{x}_{n}(t), n \in \mathbb{N}\right\}\right)\right) \leq \frac{T+4}{2}\|g\|_{L^{1}} \mathcal{S} .
$$

Since $\mu(\mathfrak{T}(\Theta \times[0,1])) \geq \mu(\Theta)$ and $\left\{q_{n}\right\}_{n} \subset \Theta$, we so get

$$
\begin{aligned}
& \sup _{t \in[0, T]}\left(\gamma\left(\left\{q_{n}(t), n \in \mathbb{N}\right\}\right)+\gamma\left(\left\{\dot{q}_{n}(t), n \in \mathbb{N}\right\}\right)\right) \\
& \quad \leq \sup _{t \in[0, T]}\left(\gamma\left(\left\{x_{n}(t), n \in \mathbb{N}\right\}\right)+\gamma\left(\left\{\dot{x}_{n}(t), n \in \mathbb{N}\right\}\right)\right)
\end{aligned}
$$

and, in view of (24) and $\left(5_{i i i}\right)$, we have that

$$
\sup _{t \in[0, T]}\left(\gamma\left(\left\{q_{n}(t), n \in \mathbb{N}\right\}\right)+\gamma\left(\left\{\dot{q}_{n}(t), n \in \mathbb{N}\right\}\right)\right)=0 .
$$

Inequality (24) implies that

$$
\sup _{t \in[0, T]}\left(\gamma\left(\left\{x_{n}(t), n \in \mathbb{N}\right\}\right)+\gamma\left(\left\{\dot{x}_{n}(t), n \in \mathbb{N}\right\}\right)\right)=0 .
$$

Now, we show that both the sequences $\left\{x_{n}\right\}$ and $\left\{\dot{x}_{n}\right\}$ are equi-continuous. Let $\tilde{\Theta} \subset E$ be such that $q_{n}(t) \in \tilde{\Theta}$ and $\dot{q}_{n}(t) \in \tilde{\Theta}$ for all $n \in \mathbb{N}$ and $t \in[0, T]$. Thus, we get that $\left\|\ddot{x}_{n}(t)\right\|=$ $\lambda_{n}\left\|f_{n}(t)\right\| \leq v_{\tilde{\Theta}}(t)$, where $v_{\tilde{\Theta}} \in L^{1}([0, T],[0, \infty))$ comes from $\left(5_{i i}\right)$, and so $\left\{\ddot{x}_{n}\right\}_{n}$ is uniformly integrable. This implies that $\left\{\dot{x}_{n}\right\}_{n}$ is equi-continuous. Moreover, according to (23), we obtain that

$$
\left\|\dot{x}_{n}(t)\right\| \leq \int_{0}^{T} v_{\tilde{\Theta}}(s) d s
$$


for all $n \in \mathbb{N}$ and $t \in[0, T]$, implying that $\left\{\dot{x}_{n}\right\}_{n}$ is bounded; consequently, also $\left\{x_{n}\right\}_{n}$ is equi-continuous. Therefore,

$$
\bmod _{C}\left(\left\{x_{n}\right\}\right)=\bmod _{C}\left(\left\{\dot{x}_{n}\right\}\right)=0 .
$$

In view of (25), we have so obtained that

$$
\mu(\mathfrak{T}(\Theta \times[0,1]))=(0,0) .
$$

Hence, also $\mu(\Theta)=(0,0)$ and since $\mu$ is regular, we have that $\Theta$ is relatively compact. Therefore, condition (iii) in Proposition 3.1 holds.

ad (iv) For all $q \in Q$, the problem $P(q, 0)$ has only the trivial solution. Since $0 \in K$, condition (iv) in Proposition 3.1 is satisfied.

ad (v) Let $q^{*} \in Q$ be a solution of the b.v.p. $P\left(q^{*}, \lambda\right)$ for some $\lambda \in(0,1)$, i.e., a fixed point of the solution mapping $\mathfrak{T}$. In view of conditions (7), (8) (see Proposition 4.1), $K$ is, for all $\lambda \in(0,1)$, a bound set for the problem

$$
\left.\begin{array}{l}
\ddot{q}_{*}(t) \in \lambda F\left(t, q^{*}(t), \dot{q}^{*}(t)\right), \quad \text { for a.a. } t \in[0, T], \\
x(T)=x(0)=0 .
\end{array}\right\}
$$

This implies that $q^{*} \notin \partial Q$, which ensures condition (v) in Proposition 3.1.

If the mapping $F(t, x, y)$ is globally u.s.c. in $(t, x, y)$ (i.e., a Marchaud map), then we are able to improve Theorem 5.1 in the following way.

Theorem 5.2 Consider the Dirichlet b.v.p. (1), where F: $[0, T] \times E \times E \multimap E$ is an upper semicontinuous mapping with compact, convex values. Assume that $K \subset E$ is an open, convex set containing 0 . Moreover, let conditions $\left(5_{i}\right),\left(5_{i i}\right),\left(5_{i i i}\right)$ from Theorem 5.1 be satisfied.

Furthermore, let there exist a function $V \in C^{1}(E, \mathbb{R})$ with a locally Lipschitz Frechét derivative $\dot{V}$ satisfying $(\mathrm{H} 1)$ and $(\mathrm{H} 2)$. Moreover, let, for all $x \in \partial K, t \in(0, T), \lambda \in(0,1)$ and $y \in E$ satisfying (11), condition (12) hold for all $w \in \lambda F(t, x, y)$. Then the Dirichlet b.v.p. (1) admits a solution whose values are located in $\bar{K}$.

Proof The verification is quite analogous as in Theorem 5.1 when just replacing the usage of Proposition 4.1 by Proposition 4.2.

\section{Illustrative example}

Example 6.1 Let $E=H$ be a Hilbert space and let us consider the Dirichlet b.v.p.

$$
\left.\begin{array}{l}
\ddot{x}(t) \in F_{1}(t, x(t), \dot{x}(t))+F_{2}(t, x(t), \dot{x}(t)), \quad \text { for a.a. } t \in[0, T], \\
x(0)=x(T)=0,
\end{array}\right\}
$$

where

(i) $F_{1}:[0, T] \times H \times H \multimap H$ is an upper-Carathéodory multivalued mapping and $F_{1}(t, \cdot, \cdot): H \times H \multimap H$ is completely continuous for a.a. $t \in[0, T]$ such that

$$
\left\|F_{1}(t, x, y)\right\| \leq v_{1}\left(t, D_{0}, D_{1}\right) \in L^{1}([0, T],[0, \infty))
$$


for a.a. $t \in[0, T]$ and all $x, y \in H$ with $\|x\| \leq D_{0},\|y\| \leq D_{1}$,

(ii) $F_{2}:[0, T] \times H \times H \multimap H$ is a Carathéodory multivalued mapping such that

$$
\left\|F_{2}(t, 0,0)\right\| \leq v_{2}(t) \in L^{1}([0, T],[0, \infty)) \quad \text { for a.a. } t \in[0, T]
$$

and $F_{2}(t, \cdot, \cdot): H \times H \multimap H$ is Lipschitzian for a.a. $t \in[0, T]$ with the Lipschitz constant

$$
L<\frac{2}{T(T+4)} .
$$

Moreover, suppose that

(iii) there exist $R>0$ and $\varepsilon>0$ such that, for all $x \in H$ with $R-\varepsilon<\|x\| \leq R, t \in(0, T)$, $y \in H, \lambda \in(0,1)$ and $w \in \lambda\left(F_{1}(t, x, y)+F_{2}(t, x, y)\right)$, we have

$$
\langle y, y\rangle+\langle x, w\rangle>0
$$

Then the Dirichlet problem (26) admits, according to Theorem 5.1, a solution $x(\cdot)$ such that $\|x(t)\| \leq R$ for all $t \in[0, T]$.

Indeed. The properties of $F_{2}$ guarantee that $F_{2}$ satisfies the inequality $(c f ., e . g .,[20])$

$$
\gamma\left(F_{2}\left(t, \Omega_{1} \times \Omega_{2}\right)\right) \leq L\left(\gamma\left(\Omega_{1}\right)+\gamma\left(\Omega_{2}\right)\right)
$$

for a.a. $t \in[0, T]$ and every bounded $\Omega_{1}, \Omega_{2} \subset H$, where $\gamma$ stands for the Hausdorff measure of noncompactness in $H$.

Since $F_{1}(t, \cdot, \cdot)$ is completely continuous and thanks to the algebraic semi-additivity of $\gamma$, inequality (27) can be rewritten into

$$
\gamma\left(F_{1}\left(t, \Omega_{1} \times \Omega_{2}\right)+F_{2}\left(t, \Omega_{1} \times \Omega_{2}\right)\right) \leq L\left(\gamma\left(\Omega_{1}\right)+\gamma\left(\Omega_{2}\right)\right)
$$

for a.a. $t \in[0, T]$ and every bounded $\Omega_{1}, \Omega_{2} \subset H$, i.e., $\left(5_{i}\right)$, for $g:=L<\frac{2}{T(T+4)}\left(c f .\left(5_{i i i}\right)\right)$.

Moreover, according to the Lipschitzianity of $F_{2}$, the following inequalities take place:

$$
d_{H}\left(F_{2}(t, x, y), 0\right) \leq d_{H}\left(F_{2}(t, x, y), F_{2}(t, 0,0)\right)+d_{H}\left(F_{2}(t, 0,0), 0\right) \leq L(\|x\|+\|y\|)+v_{2}(t)
$$

for a.a. $t \in[0, T]$ and all $x, y \in H$.

Thus, for $\|x\| \leq D_{0},\|y\| \leq D_{1}$, we arrive at

$$
\left\|F_{1}(t, x, y)+F_{2}(t, x, y)\right\| \leq L\left(D_{0}+D_{1}\right)+v_{1}\left(t, D_{0}, D_{1}\right)+v_{2}(t):=v_{\Omega}(t) \in L^{1}([0, T],[0, \infty)),
$$

i.e., (18) in $\left(5_{i i}\right)$.

Finally, in view of Remark 4.1, we can define the bounding function $V \in C^{2}(H, \mathbb{R})$ by the formula

$$
V(x):=\frac{1}{2}\left(\langle x, x\rangle-R^{2}\right)
$$

and the bound set $K$ as $K:=\{x \in H \mid\|x\|<R\}$ in order to get a claim. 
Remark 6.1 Consider again (26) in a Hilbert space $H$, but let this time $F_{1}, F_{2}$ be globally u.s.c. mappings with compact, convex values $\left(\Rightarrow F_{2}([0, T], 0,0)\right.$ is compact (cf., e.g., $[15$, Proposition I.3.20]) and, in particular, bounded) such that

(i) $\quad F_{1}(t, \cdot, \cdot): H \times H \multimap H$ is a completely continuous mapping for a.a. $t \in[0, T]$ such that

$$
\left\|F_{1}(t, x, y)\right\| \leq v_{1}\left(t, D_{0}, D_{1}\right) \in L^{1}([0, T],[0, \infty))
$$

for a.a. $t \in[0, T]$ and all $x, y \in H$ with $\|x\| \leq D_{0},\|y\| \leq D_{1}$.

(ii) $\quad F_{2}(t, \cdot, \cdot): H \times H \multimap H$ is a Lipschitzian mapping for a.a. $t \in[0, T]$ with the Lipschitz constant

$$
L<\frac{2}{T(T+4)}
$$

(iii ${ }^{u s c}$ ) There exists $R>0$ such that, for all $x \in H$ with $\|x\|=R, t \in(0, T), y \in H$ satisfying $\langle x, y\rangle=0, \lambda \in(0,1)$ and $w \in \lambda\left(F_{1}(t, x, y)+F_{2}(t, x, y)\right)$, we have

$$
\langle y, y\rangle+\langle x, w\rangle>0 .
$$

Applying now Theorem 5.2, by the analogous arguments as in Example 6.1, the Dirichlet problem (26) admits a solution $x(\cdot)$ such that $\|x(t)\| \leq R$ for all $t \in[0, T]$.

Remark 6.2 Since the solution derivative $\dot{x}(\cdot)$ takes the form

$$
\dot{x}(t) \in \int_{0}^{T} \frac{\partial}{\partial t} G(t, s)\left[F_{1}(s, x(s), \dot{x}(s))+F_{2}(s, x(s), \dot{x}(s))\right] d s,
$$

where

$$
\frac{\partial}{\partial t} G(t, s)= \begin{cases}\frac{(s-T)}{T} & \text { for all } 0 \leq t \leq s \leq T \\ \frac{s}{T} & \text { for all } 0 \leq s \leq t \leq T\end{cases}
$$

and so $\left|\frac{\partial}{\partial t} G(t, s)\right| \leq 1$ for all $t, s \in[0, T]$, we obtain (under the above assumptions) the implicit inequality

$$
D_{1} \leq \frac{1}{1-L T}\left[\int_{0}^{T} v_{1}\left(t, R, D_{1}\right) d t+\int_{0}^{T} v_{2}(t) d t+L R T\right] \text { for all } t \in[0, T],
$$

for $D_{1}:=\max _{t \in[0, T]}\|\dot{x}(t)\|$.

Thus, for $F_{1}(t, x, y) \equiv F_{1}(t, x)$, we have $v_{1}\left(t, R, D_{1}\right) \equiv v_{1}(t, R)$, and subsequently

$$
\|\dot{x}(t)\| \leq \frac{1}{1-L T}\left[\int_{0}^{T} v_{1}(t, R) d t+\int_{0}^{T} v_{2}(t) d t+L R T\right] \quad \text { for all } t \in[0, T] .
$$

Similarly, if $F_{1}:[0, T] \times H \times H \mapsto H$ is compact, then

$$
\int_{0}^{T} v_{1}\left(t, R, D_{1}\right) d t \leq C_{1} T
$$


holds with a suitable constant $C_{1} \geq\left\|F_{1}(t, x, y)\right\|$, and the following estimate holds:

$$
\|\dot{x}(t)\| \leq \frac{1}{1-L T}\left[C_{1} T+L R T+\int_{0}^{T} \nu_{2}(t) d t\right] \text { for all } t \in[0, T]
$$

Because of the Dirichlet boundary conditions $x(0)=x(T)=0$ for $H=R$, there exists a zero point $t_{0} \in[0, T]$ of $\dot{x}(\cdot)$, i.e., $\dot{x}\left(t_{0}\right)=0$, by which the same estimates can be also obtained without an explicit usage of the Green function above. Otherwise, it is not so easy to obtain such estimates, because Rolle's theorem fails in general.

For obtaining the estimation of the solution derivative $\dot{x}(\cdot)$ in a Hilbert space $H$, one can also apply, under natural assumptions, the $p$-Nagumo condition derived in [7].

Competing interests

The authors declare that they have no competing interests.

Authors' contributions

All authors contributed equally in this article. They read and approved the final manuscript.

\section{Author details}

${ }^{1}$ Department of Mathematical Analysis, Faculty of Science, Palacký University, 17. listopadu 12, Olomouc, 771 46, Czech Republic. ${ }^{2}$ Department of Engineering Sciences and Methods, University of Modena and Reggio Emilia, Via G. Amendola, 2 - pad. Morselli, Reggio Emilia, I-42122, Italy.

\section{Acknowledgements}

The first and third authors were supported by the grant PrF_2012_017. The second author was supported by the national research project PRIN "Ordinary Differential Equations and Applications".

\section{Endnote}

a The m.n.c. $\bmod _{C}(\Omega)$ is monotone, nonsingular and algebraically subadditive on $C([0, T], E)(c f .$, e.g., $[20])$ and it is equal to zero if and only if all the elements $x \in \Omega$ are equi-continuous.

\section{Received: 19 October 2012 Accepted: 16 January 2013 Published: 11 February 2013}

\section{References}

1. De Blasi, FS, Pianigiani, G: Solution sets of boundary value problems for nonconvex differential inclusions. Topol. Methods Nonlinear Anal. 1, 303-314 (1993)

2. Kožušníková, M: A bounding functions approach to multivalued Dirichlet problem. Atti Semin. Mat. Fis. Univ. Modena Reggio Emilia 55, 133-151 (2007)

3. Miklaszewski, D: The two-point problem for nonlinear ordinary differential equations and differential inclusions. Univ. lagell Acta Math. 36, 127-132 (1998)

4. Pavlačková, M: A bound sets technique for Dirichlet problem with an upper-Carathéodory right-hand side. Acta Univ. Palacki. Olomuc., Fac. Rerum Nat., Math. 49(2), 95-106 (2010)

5. Lakshmikantham, V, Chandra, J, Mitchell, AR: Existence of solutions of boundary value problems for nonlinear second order systems in Banach space. Nonlinear Anal. 2, 157-168 (1978)

6. Khrennikov, AY: Dirichlet's problem in Banach space. Math. Notes Acad. Sci. USSR 34(4), 804-808 (1983)

7. Mawhin, J: Two point boundary value problems for nonlinear second order differential equations in Hilbert spaces. Tohoku Math. J. 32(2), 225-233 (1980)

8. Mönch, $\mathrm{H}$ : Boundary value problems for nonlinear differential equations of second order in Banach spaces. Nonlinear Anal. 4(5), 985-999 (1980)

9. Palmucci, M, Papalini, F: Periodic and boundary value problems for second order differential inclusions. J. Appl. Math. Stoch. Anal. 14, 161-182 (2001)

10. Schmitt, K, Volkmann, P: Boundary value problems for second order differential equations in convex subsets in a Banach space. Trans. Am. Math. Soc. 218, 397-405 (1976)

11. Zhou, WX, Peng, J: Existence of solution to a second-order boundary value problem via noncompactness measures. Discrete Dyn. Nat. Soc. 2012, Article ID 786404 (2012). doi:10.1155/2012/786404

12. Deimling, K: Multivalued Differential Equations. de Gruyter, Berlin (1992)

13. Wang, Z, Zhang, F: Two points boundary value problems in Banach spaces. Appl. Math. Mech. 17(3), 275-280 (1996) English edition

14. Gaines, RE, Mawhin, JL: Coincidence Degree and Nonlinear Differential Equations. Lect. Notes in Math., vol. 568 Springer, Berlin (1977)

15. Andres, J, Górniewicz, L: Topological Fixed Point Principles for Boundary Value Problems. Kluwer, Dordrecht (2003)

16. Andres, J, Malaguti, L, Pavlačková, M: On second-order boundary value problems in Banach spaces: a bound sets approach. Topol. Methods Nonlinear Anal. 37(2), 303-341 (2011)

17. Andres, J, Malaguti, L, Pavlačková, M: A Scorza-Dragoni approach to second-order boundary value problems in abstract spaces. Appl. Math. Inf. Sci. 6(2), 177-192 (2012) 
18. Andres, J, Malaguti, L, Taddei, V: On boundary value problems in Banach spaces. Dyn. Syst. Appl. 18, 275-302 (2009)

19. Papageorgiou, NS, Kyritsi-Yiallourou, ST: Handbook of Applied Analysis. Springer, Berlin (2009)

20. Kamenskii, MI, Obukhovskii, W, Zecca, P: Condensing Multivalued Maps and Semilinear Differential Inclusions in Banach Spaces. de Gruyter, Berlin (2001)

21. Hu, S, Papageorgiou, NS: Handbook of Multivalued Analysis, Vol. I: Theory. Kluwer, Dordrecht (1997)

22. Vrabie, II: Compactness Methods for Nonlinear Evolutions, 2nd edn. Longman, Harlow (1990)

23. Andres, J, Kožušníková, M, Malaguti, L: Bound sets approach to boundary value problems for vector second-order differential inclusions. Nonlinear Anal. 71(1-2), 28-44 (2009)

24. Aubin, JP, Cellina, A: Differential Inclusions. Springer, Berlin (1984)

doi:10.1186/1687-2770-2013-25

Cite this article as: Andres et al.: Dirichlet problem in Banach spaces: the bound sets approach. Boundary Value Problems 2013 2013:25.

\section{Submit your manuscript to a SpringerOpen ${ }^{0}$ journal and benefit from:}

- Convenient online submission

- Rigorous peer review

- Immediate publication on acceptance

- Open access: articles freely available online

- High visibility within the field

- Retaining the copyright to your article 